\title{
Value of turbinoplasty in rhinosurgery - a controlled randomized study*
}

\author{
Fabian Sommer, Marc Oliver Scheithauer, Thomas Karl Hoffmann, \\ Anna-Sophia Grossi, Kerstin Hauck, Jörg Lindemann
}

Department of Otorhinolaryngology, Head and Neck Surgery, University Hospital Ulm, Frauensteige 12, 89075 Ulm, Germany
Rhinology 57: 5, $352-357,2019$

https://doi.org/10.4193/Rhin19.084

*Received for publication:

February 6, 2019

Accepted: May 3, 2019

\begin{abstract}
Background: Turbinoplasty is a common procedure in patients with nasal obstruction and hypertrophy of the nasal turbinates. A general recommendation regarding the necessity of turbinoplasty in functional rhinosurgery is still missing. For the first time, the value of turbinoplasty in septo- and septorhinoplasty regarding patient satisfaction as well as objective data in rhinomanometry and acoustic rhinometry was analyzed in a prospective randomized controlled trial.
\end{abstract}

Methodology: 73 patients with nasal obstruction due to septal deviation and / or a deviated nose were included in the study. After randomization, anterior turbinoplasty was or was not performed during septo- or septorhinoplasty. Pre- and postoperative rhinomanometry and acoustic rhinometry were accomplished. NOSE ${ }^{\odot}$ and SNOT 20 questionnaires were completed by the patients before and 9 months after surgery. Additionally, the patients were asked about their subjective satisfaction.

Results: $81 \%$ of the patients were subjectively satisfied with the postoperative improvement of nasal breathing. There was a significant improvement in the values of $\operatorname{NOSE}^{\circ}$ and SNOT 20 questionnaires with no relevant difference between the two study groups. Acoustic rhinometry and rhinomanometry showed values in MCA 1 and 2, Vol 1 and Vol 2 as well as higher nasal flows with no statistically significant difference between the two study groups.

Conclusions: Patient satisfaction after functional septo- and septorhinoplasty is high and does not seem to be affected by turbinate surgery. There was no statistically significant difference in the postoperative results regarding objective rhinological measurements with or without turbinoplasty. As extensive resections of the turbinates can have a negative impact on nasal physiology, the indication for turbinoplasty must be considered carefully.

Key words: turbinoplasty, nasal breathing, rhinosurgery, septoplasty, septorhinoplasty

\section{Introduction}

The nasal turbinates enlarge the mucosal surface and ensure a close contact of inhaled air and the mucosa. This allows the most efficient heating and humidification possible. However, turbinoplasty is often used as an adjunct to septo- and septorhinoplasty. Hypertrophy of the nasal turbinates has many reasons. Allergic predisposition, recurrent infections, dry nasal mucosa due to long lasting exposure to conditioned air and an abuse of nasal decongestants are just a few or the manifold reasons. Frequently there is a compensatory hypertrophy of the turbinates as a result of septal deviation.
In 2016, 101.799 submucosal resections and plastic reconstructions of the nasal septum and 158.778 procedures regarding the inferior turbinate were registered just in Germany. There are many different ways to reduce the volume of the inferior turbinate described in the current literature ${ }^{(1-10)}$. Laser application, radiofrequency ablation, bipolar coagulation, partial resection, pyriform turbinoplasty and the anterior inferior turbinoplasty are just a few procedures commonly used to supplement septoplasty or septorhinoplasty. Many patients report an initial improvement in nasal breathing after turbinoplasty. In the long term, however, a frequent recurrence of complaints is reported, 
especially after laser application (1).

The "anterior inferior turbinoplasty" is considered as one of the moderate procedures that reduces the volume of the inferior turbinate without over-resecting its mucosal surface. Here, the mucosa is dissected from the bone via a cut on the lower edge of the head of the inferior turbinate. Subsequently, the anteriorinferior part of the turbinate bone and the associated submucosal tissue as well as the lateral part of the mucosa are removed. Subsequently, the medial tissue and mucosa is lateralized and the shape of the head of the turbinate as well as and large parts of the mucosa are preserved.

Also, from a physiological point of view the nasal turbinates are able to significantly increase and reduce their volume during the nasal cycle due to the high density of venous vessels within the tissue. Within the nasal cycle activity and regeneration phases alternate allowing for greater exposure of one turbinate and concomitant regeneration of the other turbinate by reduced exposure to inhaled air ${ }^{(11-14)}$. Long-term observations suggest that the turbinate is able to adapt to the shape of the septum ${ }^{(10)}$. The hypothesis suggests that even after turbinoplasty, a long-term recurrent hypertrophy of the turbinate leads to an adaptation to the anatomical configuration of the septum in order to restore the physiological bottlenecks. This is often seen in strongly deviated septal configurations, where the inferior turbinate at the concave side of the septum hypertrophies and adapts its form to the septum. Of course, there are indications for a turbinoplasty as there are severe septal deviations with consecutive hypertrophy of the inferior turbinate that require a volume reduction in order to gain space for the corrected septum. Additionally, surgical resection of the turbinates increases the risk of postoperative bleeding and often requires nasal packing.

However, in many cases a turbinoplasty is routinely performed in the context of a septo- and septorhinoplasty. The usefulness of a routinely performed turbinoplasty in septo- and septorhinoplasty can be questioned considering the adaptability of the nasal turbinates. So far, there are no studies in the current literature analyzing the long-term effects of turbinoplasty as a supplement in septoplasty and septorhinoplasty in a randomized controlled setting. Therefore, the aim of the present study was the analysis of the value of turbinoplasty in this context in a prospective, controlled, randomized trial.

\section{Materials and methods}

A prospective, controlled randomized study was performed monocentrically at the Department of Otorhinolaryngology, Head and Neck Surgery of the University Hospital UIm. The study was approved by the local ethics committee in 2015 (No 326/15). Patients with septal deviation or a functionally relevant deviated nose undergoing septo- or septorhinoplasty for functional reasons were included in the study.
The first study group received a regular septoplasty / functional septorhinoplasty without any manipulation regarding the turbinates (study group "NO TPL").

In patients of the second group, in addition to the septoplasty / functional septorhinoplasty, bilateral anterior inferior turbinoplasty was performed (study group "TPL"). In order to receive comparable results, an anterior inferior turbinoplasty was performed in all patients belonging to the TPL group. Here, an incision is made on the caudal edge of the head of the lower turbinate and a medial mucosal flap is formed. The caudal parts of the turbinate bone including the lateral submucosal swelling parts are resected and then the medial mucosal flap is replaced. The advantage of this method is the only minor reduction of the mucosal surface and the sparing of the head of the lower turbinate as part of the nasal valve.

The procedures were performed by four different senior physicians. To ensure comparable results, the turbinoplasty was performed by all surgeons in the exact same technique. The patients were randomized to one of the two groups. Patients were not told which of the two groups they belonged to until the last examination and questioning nine months postoperatively. There were 3 examinations for each patient. Preoperatively, the SNOT20 (GAV) and the NOSE ${ }^{\odot}$ questionnaires were completed by all participants. Nasal endoscopy with a digital photo documentation of the septum and the inferior turbinates, rhinometry and rhinomanometry were performed using Rhino-Sys (Happersberger, otopront $\mathrm{GmbH}$, Germany) according to the consensus report on acoustic rhinometry and rhinomanometry ${ }^{(15)}$. At the end of their inpatient stay, the study participants received a modified "Postoperative Questionnaire" in order to measure acute postoperative complaints. This questionnaire covered complaints of nasal breathing, pressure in the nose, problems sleeping and crusting in the nose on a scale from 1 (minor complaints) to 4 (severe complaints).

The third and final examination was performed nine months postoperatively. Each of the study participants completed in the SNOT20 (GAV) and NOSE $^{\odot}$ for the second time. Additionally, a postoperative feedback was captured by questionnaire. Rhinomanometry and acoustic rhinometry were repeated as well as the endoscopy and the intranasal photo documentation of both nasal cavities.

Exclusion criteria were any kind of previous surgeries regarding the nose, turbinates and the paranasal sinuses, patients with obstructive sleep apnoea, patients with regular cigarette consumption and patients of more than 60 or less than 18 years of age. Patients eligible for study enrollment were specifically asked for allergic complaints. In all patients a prick test was performed to exclude cutaneous sensitization to inhaled allergens. Patients with an allergic anamnesis or with a positive prick test were excluded from the study.

The collected data was anonymized using a unique identifica- 
Table 1. Pre- and postoperative results of rhinomanometry.

\begin{tabular}{|lcc|}
\hline \multicolumn{2}{|c|}{$\begin{array}{c}\text { Rhinomanometry (bilateral mean inspiratory flow) } \\
\text { Average [ml/s] }\end{array}$} \\
\hline \multirow{2}{*}{ NOTPL } & preoperative & 499.37 \\
& postoperative & 515.33 \\
TPL & preoperative & 502.25 \\
& postoperative & 594.38 \\
\hline
\end{tabular}

tion number (ID). All data was recorded with Microsoft Excel. Statistical analysis was performed by IBM SPSS Statistics data editor.

\section{Results}

73 patients were included in the study. The questionnaires from 58 patients could be included in the analysis. 51 patients returned to the final examination after 9 months. 15 patients did not return the questionnaires and did not return to hospital for the final examination. The reasons were moving the apartment, schedule difficulties or contact problems. 19 of the included patients received a septoplasty. 9 of them were additionally treated with an anterior inferior turbinoplasty. 54 patients received a septorhinoplasty due to a deviated nose. 28 of them received an anterior inferior turbinoplasty. The age and gender distribution of the two study groups was comparable.

47 of 58 patients ( $81 \%$ ) were satisfied with the general results of surgery regarding improved nasal breathing and would opt for surgery again.

\section{Rhinomanometry}

The mean inspiratory flow was $499 \mathrm{ml} / \mathrm{s}$ preoperatively vs. 515 $\mathrm{ml} / \mathrm{s}$ postoperatively in the NO TPL group and $502 \mathrm{ml} / \mathrm{s}$ preoperatively vs. $594 \mathrm{ml} / \mathrm{s}$ in the TPL group. Table 1 shows the results of inspiratory flow $(\mathrm{ml} / \mathrm{s}, 150 \mathrm{~Pa})$ in rhinomanometry, Figure 1 depicts the changes in a box plot. There was no statistically significant difference between the two groups (TPL vs. NO TPL) preoperatively $(p=0.724)$ and postoperatively $(p=0.122)$. Comparing the pre- and postoperative results of the patients, there was a statistically significant difference $(p=0.004)$.

\section{Acoustic rhinometry}

The difference in MCA2 averaged $0.30 \mathrm{~cm}^{2}$ preoperatively vs. $0.83 \mathrm{~cm}^{2}$ postoperatively in the NO TPL group and $0.25 \mathrm{~cm}^{2}$ preoperatively vs. $0.91 \mathrm{~cm}^{2}$ postoperatively in the TPL-group. A statistically significant difference was detected between the pre- and postoperative result within the groups (NOTPL: $p=$ 0.002 , TPL: $p=0.0001$ ) with regard to MCA2, but no significant difference between the groups TPL and NO TPL, preoperatively $(p=0.064)$ and postoperatively $(p=0.828)$. Table 2 depicts

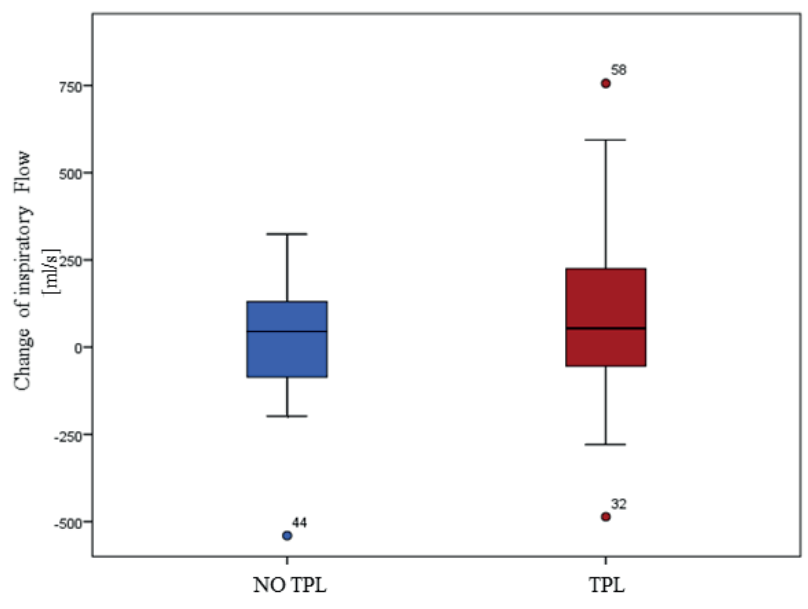

Figure 1. Box Plot of the flow-change in rhinomanometry (comparing the turbinoplasty group and the no turbinoplasty group) in $\mathrm{ml}$.

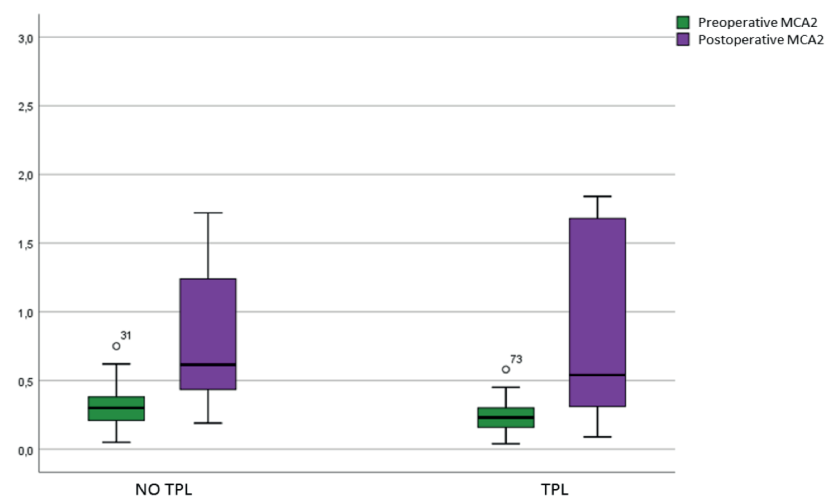

Figure 2. Box Plot of the pre- and postoperative acoustic rhinometry (MCA2, comparing the TPL and the NO TPL group).

the change in acoustic rhinometry regarding MCA2. Figure 2 displays the results of the pre- and postoperative acoustic rhinometry (MCA2) in a box blot.

\section{Questionnaires}

The SNOT 20 (GAV) showed an average total score of 26 points preoperatively vs. 16 points postoperatively in the NO TPL-group. The average score decreased from 30 points to 16 points on average in the TPL-group. The pre- and postoperative difference was statistically significant $(p<0.05)$ for both groups regardless of whether a turbinoplasty was performed or not. The same result was detected for the $\operatorname{NOSE}^{\oplus}$ questionnaire $(p<0.05)$. Here the average score decreased from 64 to 28 for the NO TPLgroup whereas the TPL-group decreased from 62 to 28 .

Analyzing the differences between the two groups, there was no significant difference between the patients of the two groups, with and without turbinoplasty $(p>0.05)$ (Tables 3 and 4). 
Table 2. Changes in MCA2.

\begin{tabular}{lll}
\multicolumn{2}{c}{$\begin{array}{c}\text { Acoustic Rhinometry: Changes in MCA2 (deviated side of the } \\
\text { septum) }\end{array}$} & $\begin{array}{c}\text { Average } \\
\text { NOTPL }\end{array}$ \\
& preoperative & $0.30 \mathrm{~cm}^{2}$ \\
TPL & postoperative & $0.83 \mathrm{~cm}^{2}$ \\
& preoperative & $0.25 \mathrm{~cm}^{2}$ \\
& postoperative & $0.91 \mathrm{~cm}^{2}$
\end{tabular}

Table 3. Pre- and postoperative values of the SNOT 20 GAV questionnaire within the groups (TPL vs. No TPL).

$\begin{array}{lll}\text { SNOT } 20 \text { GAV questionnaire } & \\ & & \text { Total score (average) } \\ \text { NOTPL } & \text { preoperative } & 26 / 100 \\ & \text { postoperative } & 16 / 100 \\ \text { TPL } & \text { preoperative } & 30 / 100 \\ & \text { postoperative } & 16 / 100\end{array}$

Table 4. Pre- and postoperative values of the NOSE@ questionnaire within the groups (TPL vs. NOTPL).

\begin{tabular}{|lcr|} 
& & \\
& & \\
& & Average points \\
& & \\
& & \\
\multirow{2}{*}{ NOTPL } & preoperative & $64 / 100$ \\
& postoperative & $28 / 100$ \\
TPL & preoperative & $62 / 100$ \\
& postoperative & $28 / 100$
\end{tabular}

\section{Discussion}

Turbinoplasty is an important surgical method in the treatment of certain nasal pathologies. In many cases a turbinoplasty is still a standard step in septo- and septorhinoplasty. Mlynski et al. demonstrated the remarkable adaptability of the turbinates to the given spatial conditions. They demonstrated, that turbinates physiologically adapt to septal deviations by hypertrophying on the concave side of the septum until the physiological cleft space between the turbinate and the septum is restored. This long-term effect is also seen after turbinoplasty. On the other hand, the turbinate is able to adapt to tight spatial conditions, just to restore this gap ${ }^{(14,16-21)}$.

In this respect, a turbinoplasty does not seem to be fundamentally necessary in all patients, as the turbinate is able to produce physiological conditions on its own. These observations led us to carry out the present study.

Hypertrophy of the inferior turbinate is a common problem in nasal obstruction. There are reasons to perform an anterior inferior turbinoplasty, especially in not on conservative therapyresponsive hypertrophy ${ }^{(22,23)}$. There are a variety of operational techniques that are described in the current literature to reduce the volume of turbinates ${ }^{(1,3,7,8,24-26)}$. Under certain conditions, these techniques offer good chances of success to significantly improve the nasal breathing of affected patients. Based on the above-mentioned studies on the adaptability of the turbinates, it seems reasonable that a turbinoplasty should not be performed as standard step in in functional rhinosurgery. Patients with allergic hypertrophy of the inferior turbinate were excluded in the present study although it is a quite common pathology. However, the treatment of choice in this case is initially medicinal and only with persistence of hypertrophy surgical. Apart from impairing nasal physiology, a turbinoplasty significantly increases the risk of bleeding ${ }^{(27)}$. In addition, a recent retrospective study found that patients receiving septum and turbinoplasty had significantly more problems than those in the control group where patients received septoplasty only ${ }^{(28)}$. Extensive resections of the inferior turbinate may lead to an empty nose syndrome, which is associated with severe reduction of the quality of life ${ }^{(29)}$.

Septoplasty and septorhinoplasty are effective methods for improving subjectively nasal breathing. $81 \%$ of the patients enrolled in the study would have the operation re-performed independently of the turbinoplasty. Although the effectiveness of septoplasty in the literature is discussed quite controversially (30), our results underline its positive impact on functional nasal breathing. The direct comparison between the TPL and NO TPL groups revealed no significant difference neither in nasal air flow volumes of rhinomanometry nor in minimal cross-sectional areas and volumes of acoustic rhinometry. In both groups nasal airflow volumes were improved postoperatively. Even though active anterior rhinomanometry offers valuable information (nasal air flow volume), there are limitations of this method ${ }^{(31)}$. According to the technique of active anterior rhinomanometry, results are values of volume depending on the active cooperation of the patient. Additionally, the results do not necessarily correlate with the patient's complaints and/or clinical findings. Therefore, as much subjective (SNOT-20 and NOSE questionnaires and a self-designed questionnaire) and objective data (rhinomanometry, acoustic rhinometry) as possible were collected in the present study in order to obtain a valid statement. As patient enrollment started in 2015 and data collection was completed in 2017, the updated agreement on nasal airway function tests of 2018 could not be fully taken into consideration ${ }^{(32)}$. In selected cases, nasal breathing can be additionally enhanced by a turbinoplasty. However, this can impair the physiological functions of humidification, warming and cleaning the inhaled air. In this respect, any surgical intervention should have as little as possible impact on the physiological conditions or attempt to restore them. 


\section{Conclusion}

Nasal turbinate surgery remains an important procedure in rhinosurgery. However, it should not be performed by default in septo- or septorhinoplasty. The present results showed no benefit for patients that received a turbinoplasty during septoor septorhinoplasty in subjective (SNOT $20 \mathrm{GAV}, \mathrm{NOSE}^{\oplus}$ questionnaire) and objective (rhinomanometry, acoustic rhinometry) values.

In this respect turbinoplasty should be avoided in the context of septoplasty and septorhinoplasty, if there is no urgent reason justifying this additional procedure. The aim of rhinosurgery must be the restoration of as physiological conditions as possible and not the maximum possible nasal breathing.

\section{Acknowledgements}

\section{NA}

\section{Author contriibution}

FS is the main author of this publication. MOS, TKH, ASG and $\mathrm{KH}$ are Co-Authors and supported the creation of this manuscript with additions and corrections. JL is the senior author of this manuscript and supported the study and the preparation of this manuscript decisively.

\section{Conflict of interest}

None of the above-mentioned authors has a conflict of interest regarding this publication.

\section{References}

1. Veit JA, Nordmann M, Dietz B, et al. Three different turbinoplasty techniques combined with septoplasty: Prospective randomized trial. Laryngoscope 2017 Feb;127(2):303-308.

2. Sommer F, Lindemann J, Scheithauer M-O, et al. Nasenmuschelchirurgie. HNO 2017; 65: 443-456.

3. Simmen D, Sommer F, Briner HR, et al. The effect of 'Pyriform Turbinoplasty' on nasal airflow using a virtual model. Rhinology 2015; 53: 242-8.

4. Chen Y-L, Tan C-T, Huang H-M. Long Term Efficacy of Microdebrider-Assisted Inferior Turbinoplasty With Lateralization for Hypertrophic Inferior Turbinates in Patients With Perennial Allergic Rhinitis Laryngoscope 2008; 118: 1270-1274.

5. Van Delden MR, Cook PR, Davis WE. Endoscopic partial inferior turbinoplasty. Otolaryngol Head Neck Surg 1999; 121 406-9.

6. Mabry RL. Inferior turbinoplasty. Arch Otolaryngol Head Neck Surg 1988; 114 1189

7. Cavaliere M, Mottola G, lemma M Comparison of the Effectiveness and Safety of Radiofrequency Turbinoplasty and Traditional Surgical Technique in Treatment of Inferior Turbinate Hypertrophy. Otolaryngol - Head Neck Surg 2005; 133 972-978.

8. Mabry RL. Inferior turbinoplasty: patient selection, technique, and long-term consequences. Otolaryngol Head Neck Surg 1988; 98: 60-6.

9. Janda P, Sroka R, Baumgartner R, et al. Laser treatment of hyperplastic inferior nasal turbinates: a review. Lasers Surg Med 2001; 28 404-13.

10. Hol MK, Huizing EH. Treatment of inferior turbinate pathology: a review and critical evaluation of the different techniques. Rhinology 2000; 38: 157-66.

11. Tahamiler R, Yener M, Canakcioglu S Detection of the nasal cycle in daily activity by remote evaluation of nasal sound.
Arch Otolaryngol Head Neck Surg 2009; 135: 137-42.

12. Sullivan CD, Garcia GJM, Frank-Ito DO, et al. Perception of better nasal patency correlates with increased mucosal cooling after surgery for nasal obstruction. Otolaryngol Head Neck Surg 2014; 150: 139-47.

13. Weber R, Keerl R, Radziwill R, et al. Videoendoscopic analysis of nasal steroid distribution. Rhinology 1999; 37: 69-73.

14. Lang C, Grützenmacher S, Mlynski B, et al. Investigating the Nasal Cycle Using Endoscopy, Rhinoresistometry, and Acoustic Rhinometry. Laryngoscope 2003: 113: 284-289.

15. Clement PAR, Gordts F, Standardisation Committee on Objective Assessment of the Nasal Airway, IRS, and ERS. Consensus report on acoustic rhinometry and rhinomanometry. Rhinology 2005; 43: 169-79.

16. Mlynski G, Grützenmacher S, Mlynski $B$, et al. Modelluntersuchungen zur Nasenmuschelchirurgie. Laryngo-RhinoOtologie 1993; 72: 614-617.

17. Mlynski G. Surgery of the Concha. Facial Plast Surg 1995; 11: 184-190.

18. Mlynski G, Grützenmacher S, Plontke S, et al. Correlation of nasal morphology and respiratory function. Rhinology 2001; 39: 197-201.

19. Grützenmacher S, Robinson DM, Gräfe K, et al. First Findings Concerning Airflow in Noses with Septal Deviation and Compensatory Turbinate Hypertrophy - A Model Study. ORL 2006; 68: 199-205.

20. Mlynski G. Surgery of the Nasal Septum. Facial Plast Surg 2006; 22: 223-229.

21. Grützenmacher S, Lang C, Mlynski R, et al. Long-term rhinoflowmetry: a new method for functional rhinologic diagnostics. Am J Rhinol; 19: 53-7.

22. Bakshi SS, Shankar Manoharan K, Gopalakrishnan S. Comparison of the long term efficacy of radiofrequency ablation and surgical turbinoplasty in inferior turbinate hypertrophy: a randomized clinical study. Acta Otolaryngol 2017; 137: 856-861.

23. Hamerschmidt R, Hamerschmidt R, Moreira
ATR, et al. Comparison of turbinoplasty surgery efficacy in patients with and without allergic rhinitis. Braz J Otorhinolaryngol 2016; 82: 131-139.

24. Gindros G, Kantas I, Balatsouras DG, et al. Comparison of ultrasound turbinate reduction, radiofrequency tissue ablation and submucosal cauterization in inferior turbinate hypertrophy. Eur Arch Otorhinolaryngol 2010; 267: 1727-33.

25. Shah AN, Brewster D, Mitzen K, et al. Radiofrequency Coblation Versus Intramural Bipolar Cautery for the Treatment of Inferior Turbinate Hypertrophy. Ann Otol Rhinol Laryngol 2015; 124: 691-697.

26. Rozsasi A, Leiacker $R$, Rettinger $G$, et al. Impact of resection of the turbinates and the lateral nasal wall on particle deposition. Laryngoscope 2004; 114: 646-651.

27. Brunworth J, Holmes J, Sindwani R Inferior Turbinate Hypertrophy: Review and Graduated Approach to Surgical Management. Am J Rhinol Allergy 2013; 27: 411-415.

28. Dąbrowska-Bień J, Skarżyński PH, Gwizdalska I, et al. Complications in septoplasty based on a large group of 5639 patients. Eur Arch Oto-Rhino-Laryngology 2018; 275: 1789-1794.

29. Scheithauer MO. Surgery of the turbinates and empty nose syndrome. GMS Curr Top Otorhinolaryngol Head Neck Surg 2010; 9: Doc03.

30. van Egmond MMHT, Rovers MM, Tillema $\mathrm{AHJ}$, et al. Septoplasty for nasal obstruction due to a deviated nasal septum in adults: a systematic review. Rhinology 2018; 56: 195-208.

31. Bermüller $C$, Kirsche $H$, Rettinger $G$, et al Diagnostic accuracy of peak nasal inspiratory flow and rhinomanometry in functional rhinosurgery. Laryngoscope 2008; 118: 605-10.

32. Vogt $K$, Bachmann-Harildstad G, Lintermann $A$, et al. The new agreement of the international RIGA consensus conference on nasal airway function tests. Rhinol J 2018; 56: $133-143$ 
33. Leone C, Teodoro C, Pelucchi A Mastropasqua B, Cavigioli G, Marazzini L, et al. Bronchial responsiveness and airway inflammation in patients with nonallergic rhinitis with eosinophilia syndrome. Allergy Clin Immunol 1997; 100:775-780.

34. Zambetti G, Ciofalo A, Romeo R, Soldo P, Fusconi M, Greco A, et al. Nasal histamine responses in nonallergic rhinitis with eosinophilic syndrome. Allergy Rhinol 2015; 6 : e94-e100.

\section{PD Dr. Fabian Sommer}

Department of Otorhinolaryngology Head and Neck Surgery University Hospital UIm Frauensteige 12 89075 Ulm Germany

Tel: +49 73150059500

Fax: +49 73150059811

E-mail:

fabian.sommer@uniklinik-ulm.de

\section{ADVERTISEMENT}
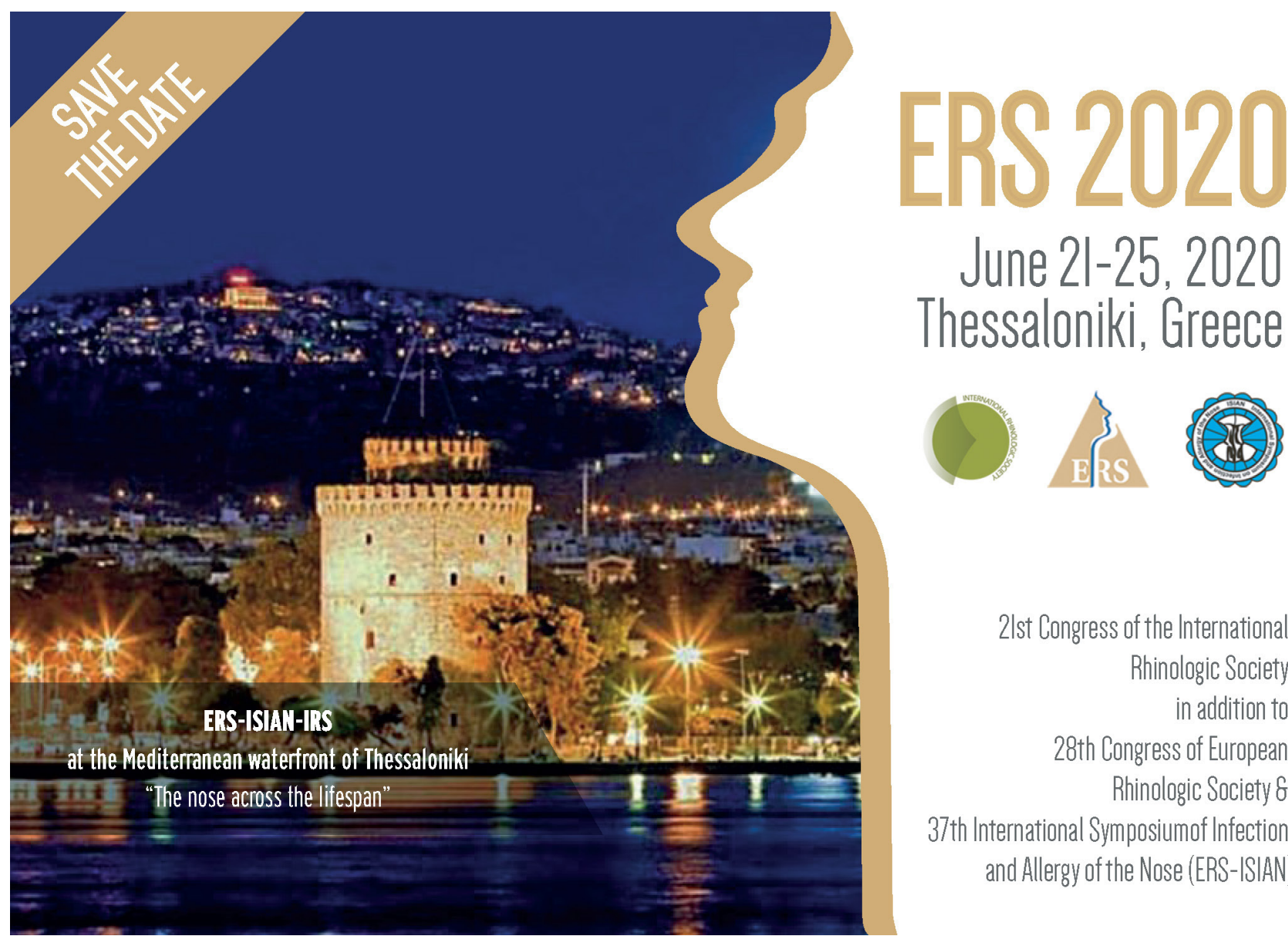

2|st Congress of the International

Rhinologic Society

in addition to

28th Congress of European

Rhinologic Society 8

37th International Symposiumof Infection and Allergy of the Nose (ERS-ISIAN) 